\title{
An Analysis of Probabilistic Forwarding of Coded Packets on Random Geometric Graphs
}

\author{
B. R. Vinay Kumar*, Navin Kashyap ${ }^{\dagger}$ \\ Indian Institute of Science \\ Bengaluru, India. \\ Email: \{vinaykb,nkashyap\}@iisc.ac.in
}

\author{
D. Yogeshwaran $\ddagger$ \\ Indian Statistical Institute \\ Bengaluru, India. \\ Email: d.yogesh@isibang.ac.in
}

\begin{abstract}
We consider the problem of energy-efficient broadcasting on dense ad-hoc networks. Ad-hoc networks are generally modeled using random geometric graphs (RGGs). Here, nodes are deployed uniformly in a square area around the origin, and any two nodes which are within Euclidean distance of 1 are assumed to be able to receive each other's broadcast. A source node at the origin encodes $k$ data packets of information into $n(>k)$ coded packets and transmits them to all its one-hop neighbors. The encoding is such that, any node that receives at least $k$ out of the $n$ coded packets can retrieve the original $k$ data packets. Every other node in the network follows a probabilistic forwarding protocol; upon reception of a previously unreceived packet, the node forwards it with probability $p$ and does nothing with probability $1-p$. We are interested in the minimum forwarding probability which ensures that a large fraction of nodes can decode the information from the source. We deem this a near-broadcast. The performance metric of interest is the expected total number of transmissions at this minimum forwarding probability, where the expectation is over both the forwarding protocol as well as the realization of the RGG. In comparison to probabilistic forwarding with no coding, our treatment of the problem indicates that, with a judicious choice of $n$, it is possible to reduce the expected total number of transmissions while ensuring a near-broadcast.
\end{abstract}

\section{INTRODUCTION}

Broadcast mechanisms on distributed ad-hoc networks (see e.g. [1], [2]) are critical for network management. In applications such as Wireless Sensor Networks (WSNs) and Internet of Things (IoT), networks are dense with the individual nodes being energy-constrained. The nodes have limited computational ability and knowledge of the network topology. Algorithms such as flooding, although being light-weight and easy to implement, give rise to unnecessary transmissions and hence are not energy efficient (see [3]).

Probabilistic forwarding as a broadcast mechanism (see e.g., [4], [5], [6]) has been proposed in the literature as an alternative to flooding. Here, each node, on receiving a packet for the first time, either forwards it to all its onehop neighbours with probability $p$ or takes no action with

\footnotetext{
* The work of B.R. Vinay Kumar was supported in part by a fellowship from the Centre for Networked Intelligence (a Cisco CSR initiative) of the Indian Institute of Science.

${ }^{\dagger}$ The work of N. Kashyap was supported in part by a Swarnajayanti Fellowship awarded by the DST, Govt of India.

${ }^{\ddagger}$ The work of D. Yogeshwaran was supported in part by SERB-MATRICS grant.
}

probability $1-p$. While this mechanism reduces the number of transmissions, reception of a packet by a network node is not guaranteed.

To improve the chances of a network node receiving a packet and to handle packet drops, we introduce coding along with probabilistic forwarding. Let us suppose that the source possesses $k_{s}$ message packets which need to be broadcast. These $k_{s}$ message packets are first encoded into $n$ coded packets such that, for some $k \geq k_{s}$, the reception of any $k$ out of the $n$ coded packets by a node, suffices to retrieve the original $k_{s}$ message packets. Examples of codes with this property are Maximum Distance Separable (MDS) codes $\left(k=k_{s}\right)$, fountain codes $\left(k=k_{s}(1+\epsilon)\right.$ for some $\left.\epsilon>0\right)$ etc. which are used in practice.

The $n$ coded packets are indexed using integers from 1 to $n$, and the source transmits each packet to all its one-hop neighbours. Every other node in the network, upon reception of a packet (say packet $\# j$ ) uses the probabilistic forwarding mechanism described above. The node ignores all subsequent receptions of packet $\# j$. Packet collisions and interference effects are neglected.

Random network models have found wide acceptance in modeling wireless ad-hoc networks. In particular, random geometric graphs (RGGs) have been used in the literature to model spatially distributed networks (see [7] and [8]). These are generated by scattering (a Poisson number of) nodes in a finite area uniformly at random and connecting nodes within a pre-specified distance. The random distribution of nodes captures the variability in the deployment of the nodes of an ad-hoc network. The distance threshold conforms to the maximum range at which a transmission from a node, with maximum power, is received reliably. A more formal description of our network setting is provided in the next section.

In this paper, we analyze the performance of the above algorithm on RGGs. In particular, we wish to find the minimum retransmission probability $p$ for which the expected fraction of nodes receiving at least $k$ out of the $n$ coded packets is close to 1 , which we deem a "near-broadcast". Here, it is to be clarified that the expectation is over both the realization of the RGG and the probabilistic forwarding protocol. This probability yields the minimum value for the expected total number of transmissions across all the network nodes needed for a near- 
broadcast. The expected total number of transmissions is taken to be a measure of the energy expenditure in the network.

In our previous work [9], we have analyzed the probabilistic forwarding mechanism described here on deterministic graphs such as trees and grids. It was found that, introducing coded packets with probabilistic forwarding, offered significant energy benefits in terms of the number of transmissions needed for a near-broadcast on well-connected graphs such as grids and other lattice structures. However, for $d$-regular trees, such energy savings were not observed. Dense RGGs show similar behaviour as grids, i.e., for an intelligently chosen value of the number of coded packets, $n$, and the minimum forwarding probability, the energy expenditure in the network is considerably lesser for a near-broadcast, when compared to the scenario of probabilistic forwarding with no coding.

In this paper, we justify these observations using rigorous methods. While the techniques used here are similar to the ones on the grid (in [9]), the additional complications due to the randomness of the underlying graph need to be addressed. Ideas from continuum percolation, ergodic theory and Palm theory are employed to circumvent these technicalities. Our method of analysis may also extend to more general broadcasting models and other point processes.

The rest of the paper is organized as follows. In Section II, we describe our network setup and formulate our problem. Section III provides the simulation results of the probabilistic forwarding algorithm on RGGs. In Section IV, we provide definitions and notations of RGGs on $\mathbb{R}^{2}$ and marked point processes (MPPs). Additionally, we state an ergodic theorem for MPPs. Section V relates probabilistic forwarding and marked point processes. Ergodic theorems on MPPs are used to obtain some key quantities. These will serve as the main ingredients in obtaining our estimates for the minimum forwarding probability and the expected total number of transmissions which are presented in Section VI. Section VII discusses some aspects related to the assumptions and our results. Due to lack of space, most of our proofs and results are provided in [10], the extended version of this paper.

\section{PROBlem FORMULATION}

\section{A. Network setup}

A random geometric graph is parametrized by the intensity $\lambda$ and the distance threshold $r$. It suffices to study them by keeping one of the parameters fixed. In our treatment, we will fix the distance parameter $r$ to be equal to 1 , and study various properties as a function of the intensity, $\lambda$.

Construct a random geometric graph $G_{m}$ with intensity $\lambda$ and distance threshold $r=1$ on $\Gamma_{m}:=\left[\frac{-m}{2}, \frac{m}{2}\right]^{2}$ as follows:

- Step 1: Sample the number of points, $N$, from a Poisson distribution with mean $\lambda \nu\left(\Gamma_{m}\right)$. Here, $\nu(\cdot)$ is the Lebesgue measure on $\mathbb{R}^{2}$. Therefore, $N \sim \operatorname{Poi}\left(\lambda m^{2}\right)$.

- Step 2: Choose points $X_{1}, X_{2}, \cdots, X_{N}$ uniformly and independently from $\Gamma_{m}$. These form the points of a Poisson point process $\Phi$ (see, e.g., [8] or [11]), and constitute the vertex set of $G_{m}$. It is often easier to think of a point process as a counting measure $\Phi:=\sum_{i} \varepsilon_{X_{i}}$, where $\varepsilon_{x}$ is the Dirac measure; for $A \subset \mathbb{R}^{2}, \varepsilon_{x}(A)=1$ if $x \in A$ and $\varepsilon_{x}(A)=0$ if $x \notin A$. Consequently, $\Phi(A)$ gives the number of points in $A$. - Step 3: Place an edge between any two vertices which are within Euclidean distance $r=1$ of each other.

To carry out probabilistic forwarding over $G_{m}$, we need to fix a source. For this, we introduce a new point at the origin $\mathbf{0}=(0,0) \in \mathbb{R}^{2}$. More specifically, a graph, $G_{m}^{\mathbf{0}}$, is created with the underlying point process $\Phi^{\mathbf{0}} \triangleq \Phi \cup\{\mathbf{0}\}$ as the vertex set, and by introducing additional edges from 0 to nodes which are within $B_{1}(\mathbf{0})$, to the edge set of $G_{m}$. Here, $B_{1}(\mathbf{0})$ (more generally, $B_{1}(\mathbf{v})$ for $\left.\mathbf{v} \in \mathbb{R}^{2}\right)$ is a closed Euclidean ball of radius 1 centered at $\mathbf{0}(\mathbf{v})$.

The inclusion of an additional point at the origin $\mathbf{0}$ means that all the probabilistic computations need to be made with respect to the Palm probability given a point at the origin. We direct the reader to [11, Ch. 1.4] for an in-depth treatment of Palm theory. Physically, the Palm probability must be interpreted as the probability conditional on the event that the origin is a point of the point process. We denote the Palm probability by $\mathbb{P}^{0}$ and the expectation with respect to it by $\mathbb{E}^{\mathbf{0}}$.

The origin here is a distinguished vertex. Broadcasts initiated from it can be received by the nodes which are present in the component of the origin only. Denote by $C_{\mathbf{0}} \equiv C_{\mathbf{0}}\left(G_{m}^{\mathbf{0}}\right)$, the set of nodes in the component of the origin in $G_{m}^{0}$. The component of the origin in $G_{m}^{\mathbf{0}}$ forms the underlying connected graph, which we denote by $G$.

\section{B. Probabilistic forwarding on $R G G$}

Equipped with the underlying network, $G$, we now describe the probabilistic forwarding algorithm on it. The source, $\mathbf{0}$, encodes $k_{s}$ message packets into $n$ coded packets and transmits it to all its one-hop neighbours. Every other node in the network follows the probabilistic forwarding protocol. A node receiving a particular packet for the first time, forwards it to all its onehop neighbours with probability $p$ and takes no action with probability $1-p$. Each packet is forwarded independently of other packets and other nodes. The node ignores all subsequent receptions of the same packet, irrespective of the decision it took at the time of first reception.

We are interested in the following scenario. Let $R_{k, n}(G)$ be the number of nodes in $C_{0}$ that receive at least $k$ out of the $n$ coded packets. We refer to these as successful receivers. We sometimes denote this by $R_{k, n}\left(G_{m}^{\mathbf{0}}\right)$ to explicitly bring out the dependence on $m$. Given a $\delta>0$, we are interested in the minimum forwarding probability $p$, such that the expected fraction of successful receivers is at least $1-\delta$. More specifically, we define

$$
p_{k, n, \delta}=\inf \left\{p \mid \mathbb{E}\left[\frac{R_{k, n}\left(G_{m}^{\mathbf{0}}\right)}{\left|C_{\mathbf{0}}\left(G_{m}^{\mathbf{0}}\right)\right|}\right] \geq 1-\delta\right\},
$$

where the expectation is over both the graph $G_{m}^{0}$ as well as the probabilistic forwarding mechanism. Note that, from our construction, $R_{k, n}(G)=R_{k, n}\left(G_{m}^{\mathbf{0}}\right) \subseteq C_{\mathbf{0}}\left(G_{m}^{\mathbf{0}}\right)$. The number of successful receivers is normalized by the total number of 
vertices in $G$, which is the same as the number of vertices within the component of the origin, $C_{\mathbf{0}}\left(G_{m}^{\mathbf{0}}\right)$.

The performance measure of interest, denoted by $\tau_{k, n, \delta}$, is the expected total number of transmissions across all nodes when the forwarding probability is set to $p_{k, n, \delta}$. Here, it should be clarified that whenever a node forwards (broadcasts) a packet to all its one-hop neighbours, it is counted as a single (simulcast) transmission. Our aim is to determine, for a given $k$ and $\delta$, how $\tau_{k, n, \delta}$ varies with $n$, and the value of $n$ at which it is minimized (if it is indeed minimized). To this end, it is necessary to first understand the behaviour of $p_{k, n, \delta}$ as a function of $n$. In subsequent sections, we will cast the probabilistic forwarding mechanism as a marked point process and use results from ergodic theory to obtain the expected value of the number of successful receivers and the overall number of transmissions.

\section{Simulation RESUlts}

Simulations were performed on an RGG generated with $m=101$ and intensity $\lambda=4.5$ and 4 . As stated before, the distance threshold parameter $r$ was set to 1 . The probabilistic forwarding mechanism was carried out with $k=20$ packets and $n$ varying from 20 to 40 . The value of $\delta$ was set to 0.1 . Twenty realizations of $G$ were generated and 10 iterations of the probabilistic forwarding mechanism was carried out on each of the realizations. The fraction of successful receivers was averaged over each iteration and realization of the graph. This was used to find the minimum forwarding probability, $p_{k, n, \delta}$, required for a near-broadcast, which is plotted in Fig. 1(a). The $p_{k, n, \delta}$ values so obtained were further used to find the expected total number of transmissions over the same realizations. The expected total number of transmissions $\tau_{k, n, \delta}$, normalized by $\lambda m^{2}$, which is the average number of points within $\Gamma_{m}$, is shown in Fig. 1(b).

Notice that the expected number of transmissions decreases initially to a minimum and then increases. The decrease indicates the benefit of introducing coding along with probabilistic forwarding. The number of coded packets, $n$, and the probability, $p_{k, n, \delta}$, corresponding to the minimum point of Fig. 1(b) are the ideal parameters for operating the network to obtain maximum energy benefits.

\section{PReliminaries}

In this section, we introduce the tools required to characterize the performance of the probabilistic forwarding algorithm. The probabilistic forwarding mechanism on the RGG is modeled using marked point processes which are described here.

\section{A. Random geometric graphs on $\mathbb{R}^{2}$}

Our approach to analyzing the probabilistic forwarding mechanism on $G$ is to relate it to the probabilistic forwarding mechanism on a RGG generated on the whole $\mathbb{R}^{2}$ plane with the origin as the source. This means that the vertex set of the RGG is a Poisson point process, $\Phi$, on $\mathbb{R}^{2}$. We refer the reader to [8] or [11] for the background needed on Poisson point processes. In particular, we use the procedure outlined

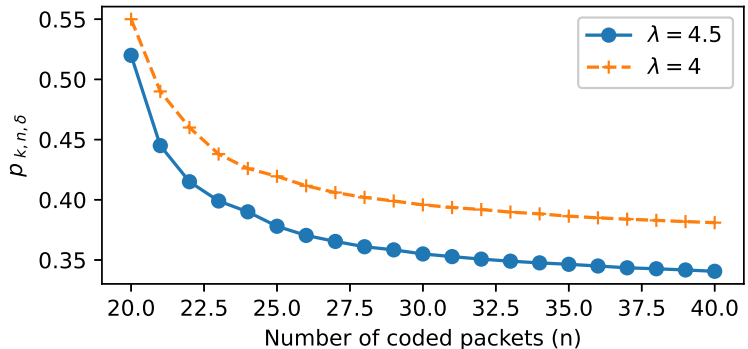

(a) Minimum retransmission probability

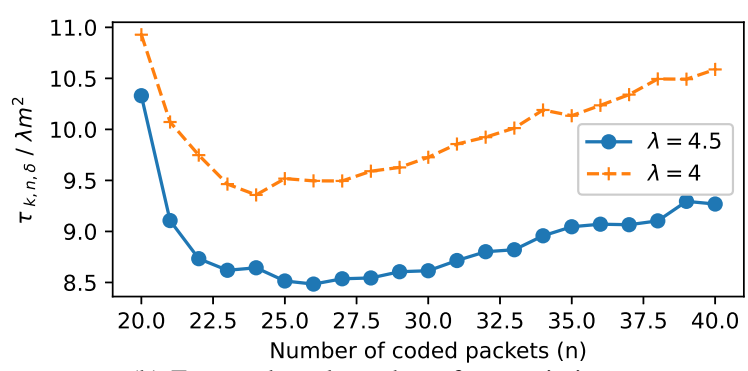

(b) Expected total number of transmission

Fig. 1: Simulations on a random geometric graph generated on $\Gamma_{101}$ with intensity $\lambda$ and distance threshold $r=1$. Probabilistic forwarding done with $k=20$ packets and $\delta=0.1$.

in $[11$, Section 1.3$]$ to construct the $R G G$ on the whole $\mathbb{R}^{2}$ plane.

Create a tiling of the $\mathbb{R}^{2}$ plane with translations of $\Gamma_{m}$, i.e., $\Gamma_{i, j}:=(i m, j m)+\Gamma_{m}$ for $i, j \in \mathbb{Z}$. On each such translation, $\Gamma_{i, j}$, construct an independent copy of a Poisson point process with intensity $\lambda$ as described in steps 1 and 2 of Section II-A. The random geometric graph $(\mathcal{G})$ is constructed by connecting vertices which are within distance 1 of each other. We then say $\mathcal{G} \sim R G G(\lambda, 1)$.

It is known that the $R G G(\lambda, 1)$ model on $\mathbb{R}^{2}$ shows a phase transition phenomenon (see [12]). For $\lambda>\lambda_{c}$, the critical intensity, there exists a unique infinite cluster, $C \equiv C(\Phi)$, in the RGG almost surely. The value of $\lambda_{c}$ is not exactly known, but simulation studies such as [13] indicate that $\lambda_{c} \approx 1.44$. The percolation probability $\theta(\lambda)$ is defined as the probability that the origin is present in the infinite cluster $C$, i.e., $\theta(\lambda):=$ $\mathbb{P}^{\mathbf{0}}(\mathbf{0} \in C)$. We remark here that there is no known analytical expression for $\theta(\lambda)$ nor are there good approximations. Since we are interested in large, dense networks, we will assume throughout our analysis that we operate in the super-critical region, i.e., $\lambda>\lambda_{c}$.

\section{B. Marked Point Process}

During the course of the probabilistic forwarding protocol on the RGG, each node decides independently whether to forward a particular packet with probability $p$. Marked point processes (MPPs) turn out to be a natural way to model such functions of an underlying point process.

Definition IV.1. Let $\Phi=\sum_{i} \varepsilon_{X_{i}}$ be a Poisson point process on $\mathbb{R}^{2}$. With each point $X_{i}$ of $\Phi$, associate a mark $Z_{i}$ taking values in some measurable space $(\mathbb{K}, \mathcal{K})$ such that $\left\{Z_{i}\right\}_{i \in \mathbb{N}} \stackrel{i i d}{\sim}$ $\Pi(\cdot)$. Then, $\tilde{\Phi}=\sum_{i} \varepsilon_{\left(X_{i}, Z_{i}\right)}$ is called an iid marked point process on $\mathbb{R}^{2} \times \mathbb{K}$ with mark distribution $\Pi(\cdot)$. 
We now state an ergodic theorem for MPPs which is used to obtain some key results required in the analysis of the probabilistic forwarding protocol in Section V.

\section{Ergodic theorem}

Let $(\Omega, \mathcal{F}, \mathbb{P})$ be the probability space over which an iid marked point process $\tilde{\Phi}=\sum_{i} \varepsilon_{\left(X_{i}, Z_{i}\right)}$ is defined with mark distribution $\Pi(\cdot)$. Let $\theta_{x}: \Omega \rightarrow \Omega$, for $x \in \mathbb{R}^{2}$, be the operator which shifts each point of $\tilde{\Phi}$ by $-x$, i.e., $\theta_{x} \tilde{\Phi}=\sum_{i} \varepsilon_{\left(X_{i}-x, Z_{i}\right)}$ and let $(\mathbb{K}, \mathcal{K})$ be the measurable space of marks. Let $f$ : $\mathbb{K} \times \Omega \rightarrow \mathbb{R}_{+}$be a non-negative function of the MPP. Then, by the ergodic theorem for marked random measures (see [14, Theorem 8.4.4]), we have $\mathbb{P}$-almost surely ( $\mathbb{P}$-a.s.),

$$
\frac{1}{\nu\left(\Gamma_{m}\right)} \sum_{X_{i} \in \Gamma_{m}} f\left(Z_{i}, \theta_{X_{i}}(\omega)\right) \stackrel{m \rightarrow \infty}{\longrightarrow} \lambda \int_{\mathbb{K}} \mathbb{E}^{(\mathbf{0}, z)}[f(z, \omega)] \Pi(d z)
$$

where $\mathbb{E}^{(0, z)}$ is the expectation with respect to the Palm probability $\mathbb{P}^{(\mathbf{0}, z)}$ conditional on the mark, $z$. If $f(z, \omega)=f(\omega)$, then (2) reduces to

$$
\frac{1}{\nu\left(\Gamma_{m}\right)} \sum_{X_{i} \in \Gamma_{m}} f\left(\theta_{X_{i}}(\omega)\right) \stackrel{m \rightarrow \infty}{\longrightarrow} \lambda \mathbb{E}^{\mathbf{0}}[f(\omega)] \quad \mathbb{P} \text {-a.s.. }
$$

\section{PRobabilistic FORWARDING AND MPPs}

In this section, we formulate probabilistic forwarding using the framework of marked point processes. Ergodic theorems for MPPs are then used to derive relevant results which will be used to obtain estimates for $p_{k, n, \delta}$ and $\tau_{k, n, \delta}$. It should be noted here that all the graphs and point processes discussed in this section are on the whole $\mathbb{R}^{2}$ plane.

\section{A. Single packet probabilistic forwarding}

Consider the probabilistic forwarding of a single packet on $\mathcal{G} \sim R G G(\Phi, 1)$ defined on a PPP $\Phi$ of intensity $\lambda$ on $\mathbb{R}^{2}$. Let $\mathcal{G}^{0}$ be the graph created with the underlying point process being $\Phi^{\mathbf{0}} \triangleq \Phi \cup\{0\}$ as the vertex set, and introducing additional edges from $\mathbf{0}$ to nodes which are within $B_{1}(\mathbf{0})$, to the edge set of $\mathcal{G}$. We assign a mark 1 to a node if it decides to transmit the packet and 0 otherwise. Thus, the mark space is $\mathbb{K}=\{0,1\}$ and $\tilde{\Phi}$ is an iid MPP with a $\operatorname{Ber}(p)$ mark distribution. Note that the origin, $\mathbf{0}$, has mark 1 since it always transmits the packet. Also, the subset of nodes which have mark 1 form a thinned point process of intensity $\lambda p$, and the subset of vertices with mark 0 form a $\lambda(1-p)$-thinned process. Denote these by $\Phi^{+}$and $\Phi^{-}$respectively, and the corresponding RGGs by $\mathcal{G}^{+}$and $\mathcal{G}^{-}$. Notice that the set of vertices of $\Phi^{+}$which are in the same cluster as the origin are the vertices which receive the packet from the source and transmit it. Thus, the number of vertices in the cluster containing the origin in $\mathcal{G}^{+}$(call this set of nodes $C_{0}^{+}$), is the number of transmissions of the packet.

In addition to the nodes of the cluster containing the origin in $\mathcal{G}^{+}$, the nodes of $\mathcal{G}^{-}$which are within distance 1 from them, also receive the packet. To account for them, we define for any cluster of nodes $S \subset \Phi^{+}$, the boundary of $S$ as

$$
\partial S=\left\{\mathbf{v} \in \Phi^{-} \mid B_{1}(\mathbf{v}) \cap S \neq \emptyset\right\},
$$

TABLE I: Results from evaluating the ergodic statements in (3) and (4) for different functions $f$.

\begin{tabular}{|c|c|cc|c|}
\hline & $f(z, \omega)$ & Result & Using \\
\hline (a) & 1 & $\frac{\Phi\left(\Gamma_{m}\right)}{\nu\left(\Gamma_{m}\right)} \stackrel{m \rightarrow \infty}{\longrightarrow} \lambda$ & $\mathbb{P}$-a.s.. & (3) \\
(b) & $z$ & $\frac{\Phi^{+}\left(\Gamma_{m}\right)}{\nu\left(\Gamma_{m}\right)} \stackrel{m \rightarrow \infty}{\longrightarrow} \lambda p$ & $\mathbb{P}$-a.s.. & (4) \\
(c) & $\mathbf{1}\{\mathbf{0} \in C\}$ & $\frac{\left|C \cap \Gamma_{m}\right|}{\nu\left(\Gamma_{m}\right)} \stackrel{m \rightarrow \infty}{\longrightarrow} \lambda \theta(\lambda)$ & $\mathbb{P}$-a.s.. & (3) \\
(d) & $\mathbf{1}\left\{\mathbf{0} \in C^{+}\right\}$ & $\frac{\left|C^{+} \cap \Gamma_{m}\right|}{\nu\left(\Gamma_{m}\right)} \stackrel{m \rightarrow \infty}{\longrightarrow} \lambda p \theta(\lambda p)$ & $\mathbb{P}$-a.s.. & (3) \\
(e) & $\mathbf{1}\left\{\mathbf{0} \in C^{\text {ext }}\right\}$ & $\frac{\left|C^{\text {ext }} \cap \Gamma_{m}\right|}{\nu\left(\Gamma_{m}\right)} \stackrel{m \rightarrow \infty}{\longrightarrow} \lambda \theta(\lambda p)$ & $\mathbb{P}$-a.s.. & (4) \\
\hline
\end{tabular}

and the extended cluster of $S$ to be $S^{\text {ext }}=S \cup \partial S$. Then, the receivers are the nodes in $C_{0}^{\text {ext }}$. We refer to this as the extended cluster of the origin.

In Section VI, we relate $C_{\mathbf{0}}^{+}$and $C_{\mathbf{0}}^{\text {ext }}$ to the infinite cluster $C^{+}:=C\left(\Phi^{+}\right)$and the infinite extended cluster (IEC), $C^{\text {ext }}$, defined as the extended cluster of $C^{+}$, respectively. Specifically, in the thermodynamic limit, the expected number of vertices in $C_{\mathbf{0}} \cap \Gamma_{m}$ (resp. $C_{\mathbf{0}}^{\text {ext }} \cap \Gamma_{m}$ ) is well-approximated by the expected number of vertices within $\Gamma_{m}$ of the infinite cluster $C^{+}$(resp., of the IEC $C^{\text {ext }}$ ) for large $m$. We use the ergodic theorem stated in Section IV-C to obtain almost sure results for the fraction of nodes within $\Gamma_{m}$ of the infinite cluster $C^{+}$and the IEC $C^{\text {ext }}$ in terms of the percolation probability $\theta(\lambda)$.

\section{B. Application of the ergodic theorem}

Specializing the statement in (2) to the probabilistic forwarding of a single packet where $\mathbb{K}=\{0,1\}$ and the marks are independent, conditional on $\Phi$, with distribution given by $\Pi(1)=1-\Pi(0)=p$, we obtain for $\mathbb{P}$-a.s.,

$$
\begin{aligned}
\frac{1}{\nu\left(\Gamma_{m}\right)} & \sum_{\substack{X_{i} \in \Gamma_{m} \\
m \rightarrow \infty}} f\left(Z_{i}, \theta_{X_{i}}(\omega)\right) \\
& \stackrel{\longrightarrow}{\longrightarrow} \lambda \mathbb{E}^{(\mathbf{0}, 1)}[f(1, \omega)]+\lambda(1-p) \mathbb{E}^{(\mathbf{0}, 0)}[f(0, \omega)] .
\end{aligned}
$$

We will now use (3) and (4) to obtain key results which will help us analyze the probabilistic forwarding of a single packet on $\mathbb{R}^{2}$. In particular, we substitute different functions $f$ in (3) and (4) to obtain the results given in Table: I. We provide these derivations in the extended version of our paper [10] due to shortage of space.

\section{Remarks:}

1) For $\lambda>\lambda_{c}$, using the dominated convergence theorem (DCT) and the reciprocal of result (a) with result (c) of Table I, we also have that

$$
\mathbb{E}\left[\frac{\left|C \cap \Gamma_{m}\right|}{\Phi\left(\Gamma_{m}\right)}\right] \stackrel{m \rightarrow \infty}{\longrightarrow} \theta(\lambda) .
$$

This means that, for large $m$, the expected fraction of vertices of the infinite cluster within $\Gamma_{m}$ is a good 


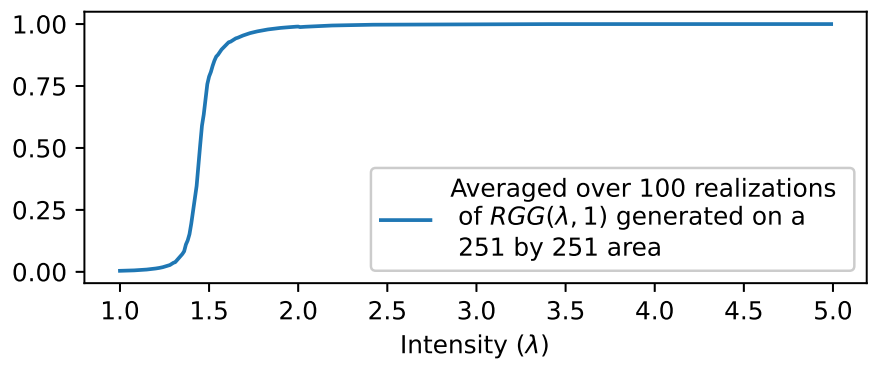

Fig. 2: Percolation probability $\theta(\lambda)$ vs. intensity $\lambda$

approximation for the percolation probability. We use this to obtain an empirical estimate of the percolation probability as follows. We generate 100 instantiations of the $R G G(\lambda, 1)$ model on $\Gamma_{251}$, for each value of $\lambda$ between 1 and 5 (in steps of 0.01 ). The fraction of vertices in the largest cluster within $\Gamma_{m}$ averaged over these instantiations is computed and taken as a proxy for the fraction of nodes in the infinite cluster within $\Gamma_{m}$. The plot obtained is shown in Fig. 2. We use the values from this plot in our numerical results.

2) In obtaining result (e) of Table I, if instead of using (4), we had substituted $f(\omega)=\mathbf{1}\left\{\mathbf{0} \in C^{\text {ext }}\right\}$ in (3), we would have obtained $\lambda \mathbb{P}^{\mathbf{0}}\left(\mathbf{0} \in C^{\text {ext }}\right)$ on the RHS. From this and (e), it is natural to define, $\theta^{\operatorname{ext}}(\lambda, p):=\mathbb{P}^{\mathbf{0}}\left(\mathbf{0} \in C^{\text {ext }}\right)=$ $\theta(\lambda p)$.

3) Comparing RHS of results (b) and (e) of Table I suggests an alternate viewpoint for the nodes that are present in the IEC. On the underlying point process $\Phi$, define new iid marks $Z^{\prime} \in \mathbb{K}=\{0,1\}$ with $\operatorname{Ber}\left(\theta^{\operatorname{ext}}(\lambda, p)\right)$ distribution. This means that a vertex is attributed mark 1 , if it is in the IEC when probabilistic forwarding is carried out with forwarding probability $p$. Then, the fraction of nodes in the IEC when marks are $Z$ corresponds to the fraction of nodes with mark 1 when marks are $Z^{\prime}$. This interpretation will be useful in proposing a heuristic approach for probabilistic forwarding of multiple packets in Section VII-B.

\section{Probabilistic forwarding of multiple packets}

Consider now the probabilistic forwarding mechanism of $n$ packets. Each node transmits a newly received packet with probability $p$ independently of other packets. It is required to find the fraction of successful receivers, the nodes that receive at least $k$ out of the $n$ packets. From our discussion of probabilistic forwarding of a single packet (in Section V-A), for large $m$, the number of nodes within $\Gamma_{m}$ that receive a packet from the origin is well-approximated by the number of nodes in the IEC. In a similar way, the fraction of successful receivers within $\Gamma_{m}$ can be well-approximated by the fraction of nodes which are present in at least $k$ out of the $n$ IECs when probabilistic forwarding is done on the $\mathrm{RGG}, \mathcal{G}^{0}$. In this subsection, we will use the ergodic theorem to obtain a limiting expression for this fraction.

Equip each vertex of the point process $\Phi$ with mark $\mathbf{Z}=$ $\left(Z_{1}, Z_{2}, \cdots, Z_{n}\right) \in \mathbb{K}=\{0,1\}^{n}$. Here the $j$-th co-ordinate of the mark represents transmission of the $j$-th packet on $\Phi$. More precisely, $Z_{j}(\cdot) \sim \operatorname{Ber}(p)$ and, for two different vertices $u$ and $v, \mathbf{Z}\left(X_{u}\right)$ and $\mathbf{Z}\left(X_{v}\right)$ are independent conditional on $\Phi$. Therefore, it forms an iid marked point process. Define $C_{k, n}^{\mathrm{ext}}$ to be the set of nodes which are present in at least $k$ out of the $n$ IECs. Taking $f(z, \omega)=\mathbf{1}\left\{\mathbf{0} \in C_{k, n}^{\text {ext }}\right\}$ in the statement of the ergodic theorem (3), we obtain, for $\mathbb{P}$ almost surely,

$$
\frac{1}{\nu\left(\Gamma_{m}\right)} \sum_{X_{i} \in \Gamma_{m}} \mathbb{1}\left\{X_{i} \in C_{k, n}^{\mathrm{ext}}\right\} \stackrel{m \rightarrow \infty}{\longrightarrow} \lambda \mathbb{P}^{\mathbf{0}}\left(\mathbf{0} \in C_{k, n}^{\mathrm{ext}}\right) .
$$

Denote by $\theta_{k, n}^{\text {ext }}(\lambda, p):=\mathbb{P}^{\mathbf{0}}\left(\mathbf{0} \in C_{k, n}^{\text {ext }}\right)$. Then the above statement reads as

$$
\lim _{m \rightarrow \infty} \frac{\left|C_{k, n}^{\text {ext }} \cap \Gamma_{m}\right|}{\nu\left(\Gamma_{m}\right)}=\lambda \theta_{k, n}^{\text {ext }}(\lambda, p) \quad \text { P-a.s.. }
$$

\section{MAIN RESULTS}

In this section, we will obtain expressions for the expected fraction of successful receivers and the expected total number of transmissions on the finite graph $G$ based on the framework that has been developed in the previous section.

While constructing $\mathcal{G}^{\mathbf{0}}$ (as described in Section V-A), the graph corresponding to $\Gamma_{0,0}$ can be taken to be $G_{m}^{\mathbf{0}}$ (with additional edges from vertices in $\Gamma_{0,0}$ to those outside it). Alternately, $G_{m}^{\mathbf{0}}$ can be constructed by considering a restriction of $\mathcal{G} \sim R G G(\lambda, 1)$ to $\Gamma_{m}$ and connecting the origin to nodes within $B_{1}(\mathbf{0})$. In essence, it is true that the distribution of nodes of $G_{m}^{\mathbf{0}}$ and $\mathcal{G}^{\mathbf{0}} \cap \Gamma_{m}$ is the same. Recall that the graph $G$ on which the probabilistic forwarding mechanism is carried out, is the component of the origin in $G_{m}^{0}$. In light of the correspondence between the vertices of $G_{m}^{\mathbf{0}}$ and $\mathcal{G}^{\mathbf{0}} \cap \Gamma_{m}$, the graph $G$ should correspond to the graph induced on the nodes within $\Gamma_{m}$ that are present in the cluster of the origin in $\mathcal{G}^{0}$. However, these nodes also include those that are contained in the cluster of the origin through paths which go outside $\Gamma_{m}$ but are not connected to the origin within $\Gamma_{m}$. We refer to these as, nodes in the cluster of the origin but without a $\Gamma_{m^{-}}$ conduit and denote them by $\widehat{C}_{\mathbf{0}, m}$. We make the following assumption about $\widehat{C}_{\mathbf{0}, m}$.

Assumption 1. $\lim _{m \rightarrow \infty} \frac{\left|\widehat{C}_{\mathbf{0}, m}\right|}{m^{2}}=0 \quad \mathbb{P}$-a.s.

The assumption can be proved to be true over a subsequence since Markov inequality accompanied by the convergence in mean (similar to $[9 \text {, Lemma VI.5 }]^{1}$ ), $\mathbb{E}\left[\frac{\left|\widehat{C}_{\mathbf{0}, m}\right|}{m^{2}}\right] \stackrel{m \rightarrow \infty}{\longrightarrow} 0$, gives convergence in probability. However, a complete proof evades us at present.

Continuing, note that $C_{\mathbf{0}}\left(\mathcal{G}^{\mathbf{0}}\right) \cap \Gamma_{m}=C_{\mathbf{0}}\left(G_{m}^{\mathbf{0}}\right) \cup \widehat{C}_{\mathbf{0}, m}$ and $C_{\mathbf{0}}\left(G_{m}^{\mathbf{0}}\right) \cap \widehat{C}_{\mathbf{0}, m}=\emptyset$. Under Assumption 1, we can also obtain the following lemma which relates the fraction of vertices in the component of the origin within $\Gamma_{m}$, to the percolation probability. The first equality stems from

\footnotetext{
${ }^{1}$ As in the case of a grid, the probability of there being a connected path in an annulus around $\Gamma_{m}$, is known to tend to 1 as $m \rightarrow \infty$ in the super-critical region, $\lambda>\lambda_{c}$.
} 
the assumption above. The proof for the second equality is provided in [10].

Lemma VI.1. Let $A=\left\{\mathbf{0} \in C\left(\mathcal{G}^{\mathbf{0}}\right)\right\}$, where $C\left(\mathcal{G}^{\mathbf{0}}\right)$ is the infinite cluster of $\mathcal{G}^{0}$. For $\lambda>\lambda_{c}$, we have

$\lim _{m \rightarrow \infty} \frac{\left|C_{\mathbf{0}}\left(G_{m}^{\mathbf{0}}\right)\right|}{\lambda m^{2}}=\lim _{m \rightarrow \infty} \frac{\left|C_{\mathbf{0}}\left(\mathcal{G}^{\mathbf{0}}\right) \cap \Gamma_{m}\right|}{\lambda m^{2}}=\theta(\lambda) \mathbf{1}_{A} \quad \mathbb{P}$-a.s.

where $C_{\mathbf{0}}\left(\mathcal{G}^{\mathbf{0}}\right)$ is the set of nodes in the cluster of the origin in $\mathcal{G}^{0}$.

Before we proceed, we recall the definition of the minimum forwarding probability in (1):

$$
p_{k, n, \delta}=\inf \left\{p \mid \mathbb{E}\left[\frac{R_{k, n}\left(G_{m}^{\mathbf{0}}\right)}{\left|C_{\mathbf{0}}\left(G_{m}^{\mathbf{0}}\right)\right|}\right] \geq 1-\delta\right\},
$$

where the expectation is over the graph as well as the probabilistic forwarding mechanism. Note that in our setting, the source, $\mathbf{0}$, always has mark 1 since it transmits all the $n$ packets. To be more explicit, define $\mathbf{1}=(1,1, \cdots, 1)$ to be the vector of all $1 \mathrm{~s}$ of length $n$. We denote by $\mathbb{E}^{(\mathbf{0 , 1})}$ the expectation with respect to the Palm probability $\mathbb{P}^{0}$ given a point at the origin, conditional on it having mark $\mathbf{Z}(\mathbf{0})=\mathbf{1}$. In terms of this, the above equation translates to

$$
p_{k, n, \delta}=\inf \left\{p \mid \mathbb{E}^{(\mathbf{0}, \mathbf{1})}\left[\frac{R_{k, n}\left(G_{m}\right)}{\left|C_{\mathbf{0}}\left(G_{m}\right)\right|}\right] \geq 1-\delta\right\} .
$$

Next, since we are addressing a broadcast problem, it is necessary that a large fraction of nodes receive a packet. This, in turn necessitates that the fraction of nodes that transmit the packet is also large. With reference to the RGG on the whole plane, this means that $\mathcal{G}^{+}$needs to have an infinite cluster. To allow for this, we make the following assumption.

Assumption 2. The forwarding probability $p$ is such that $\lambda p>$ $\lambda_{c}$.

Notice that the $p_{k, n, \delta}$ values obtained from simulations in Fig. 1 conform to this assumption. The assumption is discussed in slightly more detail in Section VII-C. We now obtain expressions for the minimum forwarding probability and the expected total number of transmissions based on these two assumptions.

\section{A. Transmissions}

Consider first the transmission of a single packet. Let $T\left(G_{m}\right)$ be the number of nodes of $G_{m}$ that receive the packet from the source and transmit it and let $\mathcal{T}(\mathcal{G}) \cap \Gamma_{m}$ be the set of nodes within $\Gamma_{m}$ that receive the packet from the source and transmit it when probabilistic forwarding is carried out on $\mathcal{G}^{2}$. From our construction, it follows that $T\left(G_{m}\right)$ is stochastically dominated by $\left|\mathcal{T}(\mathcal{G}) \cap \Gamma_{m}\right|$ since there might be nodes which receive a packet from outside $\Gamma_{m}$ and transmit it. However, it can be shown that

$$
\lim _{m \rightarrow \infty} \frac{\mathbb{E}^{(\mathbf{0}, 1)}\left[T\left(G_{m}\right)\right]}{m^{2}}=\lim _{m \rightarrow \infty} \frac{\mathbb{E}^{(\mathbf{0}, 1)}\left[\left|\mathcal{T}(\mathcal{G}) \cap \Gamma_{m}\right|\right]}{m^{2}} .
$$

${ }^{2}$ It is implicit from the use of Palm probabilities that the origin is the source and probabilistic forwarding is formulated as an MPP as described in Section V-A.
This is because the expected fraction of transmitting nodes with no $\Gamma_{m}$-conduits diminishes as $m \rightarrow \infty$. Thus, it suffices to evaluate $\lim _{m \rightarrow \infty} \frac{\mathbb{E}^{(0,1)}\left[\left|\mathcal{T}(\mathcal{G}) \cap \Gamma_{m}\right|\right]}{m^{2}}$ to find the expected number of transmissions for a single packet.

In the jargon of marked point processes, $\mathcal{T}(\mathcal{G})$ is the set of vertices with mark $Z(\cdot)=1$ that are in the cluster containing the origin. Note that the origin has mark 1 , since it always transmits the packet. As the vertices with mark 1 form a thinned point process, $\Phi^{+}$of intensity $\lambda p, \mathcal{T}(\mathcal{G})$ is the set of nodes in the cluster containing the origin in $\mathcal{G}^{+}$. In Section $\mathrm{V}-\mathrm{A}$, we denoted this set by $C_{0}^{+}$. From Assumption 2, the graph on $\Phi^{+}$is in the super-critical regime and thus possesses a unique infinite cluster, $C^{+}$. The following theorem provides the expected size of $C_{0}^{+} \cap \Gamma_{m}$. The proof proceeds by relating it to the expected size of $C^{+} \cap \Gamma_{m}$ and using the ergodic result (d) of Table I. The complete proof is provided in [10].

Theorem VI.2. For $\lambda p>\lambda_{c}$, we have

$$
\lim _{m \rightarrow \infty} \mathbb{E}^{(\mathbf{0}, 1)}\left[\frac{\left|C_{\mathbf{0}}^{+} \cap \Gamma_{m}\right|}{\lambda m^{2}}\right]=p \quad \theta(\lambda p)^{2} .
$$

Therefore, for large values of $m$, the expected number of transmissions, $\mathbb{E}^{(\mathbf{0}, \mathbf{1})}\left[T\left(G_{m}\right)\right]$, can be approximated by

$$
\mathbb{E}^{(\mathbf{0}, 1)}\left[\left|C_{\mathbf{0}}^{+} \cap \Gamma_{m}\right|\right] \approx m^{2} \lambda p \theta(\lambda p)^{2} .
$$

Consider now the transmission of multiple packets. The $n$ coded packets are transmitted independently of each other. The expected total number of transmissions of all $n$ packets would just be $n$ times the expected transmissions of a single packet. Therefore, from Theorem VI.2, we then obtain

$$
\tau_{k, n, \delta} \approx n m^{2} \lambda p_{k, n, \delta}\left(\theta\left(\lambda p_{k, n, \delta}\right)\right)^{2} .
$$

\section{B. Minimum forwarding probability}

In this section, we will obtain an expression for the minimum forwarding probability. Recall that this entails estimating $\mathbb{E}^{(\mathbf{0}, \mathbf{1})}\left[\frac{R_{k, n}\left(G_{m}\right)}{\left|C_{\mathbf{0}}\left(G_{m}\right)\right|}\right]$, where $C_{\mathbf{0}}\left(G_{m}\right)$ is the set of nodes in the component of the origin in the underlying RGG on $\Gamma_{m}$ and $R_{k, n}\left(G_{m}\right)$ are the number of nodes that receive at least $k$ out of the $n$ packets from the origin, which is the source. With reference to the discussion prior to Assumption 1, $C_{\mathbf{0}}\left(G_{m}\right)$ can be viewed as the set of nodes in the component of the origin in $\mathcal{G}^{0}$ restricted to $\Gamma_{m}$ but with only those nodes which are connected to the origin via $\Gamma_{m}$-conduits. $R_{k, n}\left(G_{m}\right)$ is the number of nodes among those in $C_{\mathbf{0}}\left(G_{m}\right)$, which are successful receivers. These arguments allow us think of the expectation $\mathbb{E}^{(\mathbf{0 , 1})}\left[\frac{R_{k, n}\left(G_{m}\right)}{\left|C_{\mathbf{0}}\left(G_{m}\right)\right|}\right]$ as being with respect to the RGG, $\mathcal{G}^{0}$, instead of the finite RGG, $G_{m}^{0}$.

Our interest is in large and dense networks, and hence it is natural to assume that the origin is part of the infinite cluster of $\mathcal{G}^{0}$. This means that the cluster of the origin in $G_{m}^{0}$ connects to the infinite cluster in $\mathcal{G}^{0}$ when $G_{m}^{0}$ is embedded within it. In other words, the event $A=\left\{\mathbf{0} \in C\left(\mathcal{G}^{0}\right)\right\}$ occurs. The results of this section are made with this assumption, which is stated below explicitly. Additional justification for this is provided in Section VII-C. 


\section{Assumption 3. The origin is part of the infinite cluster of $\mathcal{G}^{0}$.}

From the discussion above and the assumption, our interest now is to estimate $\mathbb{E}_{A}^{(\mathbf{0}, \mathbf{1})}\left[\frac{R_{k, n}\left(G_{m}\right)}{\left|C_{\mathbf{0}}\left(G_{m}\right)\right|}\right]$. The subscript $A$ in the expectation $\mathbb{E}_{A}^{(\mathbf{0 , 1})}$ indicates conditional expectation given that the event $A$ occurs. From Assumption 2, it is clear that such a conditioning can indeed be done, since $\mathbb{P}(A)=\theta(\lambda)>0$.

The following theorem gives the expected value of the fraction of successful receivers in the limit as $m \rightarrow \infty$ given the event $A$. Before we state the theorem, recall the formulation of probabilistic forwarding as a marked point process in Section V. $C_{k, n}^{\text {ext }}$ was defined as the set of nodes which are present in at least $k$ out of the $n$ IECs. Let $\theta_{k, n}^{\text {ext }} \equiv \theta_{k, n}^{\text {ext }}(\lambda, p)=\mathbb{P}^{\mathbf{0}}\left(\mathbf{0} \in C_{k, n}^{\text {ext }}\right)$. Additionally, define $A_{[t]}^{\text {ext }}$ to be the event that the origin is present only in the IECs corresponding to the packets $1,2, \cdots, t$.

Theorem VI.3. For $\lambda p>\lambda_{c}$, we have

$\lim _{m \rightarrow \infty} \mathbb{E}_{A}^{(\mathbf{0}, \mathbf{1})}\left[\frac{R_{k, n}\left(G_{m}\right)}{\left|C_{\mathbf{0}}\left(G_{m}\right)\right|}\right]=\frac{1}{\theta(\lambda)^{2}} \sum_{t=k}^{n}\left(\begin{array}{l}n \\ t\end{array}\right) \theta_{k, t}^{e x t} \mathbb{P}^{(\mathbf{0}, \mathbf{1})}\left(A_{[t]}^{\text {ext }}\right)$

The proof is on similar lines as that on the grid in [9]. It relies on carefully relating the fraction of successful receivers on $G$ to the fraction of nodes present in at least $k$ out of the $n$ IECs corresponding to probabilistic forwarding on $\mathcal{G}^{0}$. An outline of the proof is given in the extended version of this paper, [10]. The following proposition is used to express $\mathbb{P}^{(\mathbf{0}, \mathbf{1})}\left(A_{[t]}^{\mathrm{ext}}\right)$ in terms of $\theta_{k, n}^{\text {ext }}$. The proof is provided in [10].

\section{Proposition VI.4.}

$$
\mathbb{P}^{(\mathbf{0 , 1})}\left(A_{[t]}^{\text {ext }}\right)=\left\{\begin{array}{ll}
\frac{\theta_{t, n}^{\text {ext }}-\theta_{t+1, n}^{\text {ext }}}{\left(\begin{array}{c}
n \\
t
\end{array}\right)} & 0 \leq t \leq n-1 \\
\theta_{n, n}^{\text {ext }} & t=n
\end{array} .\right.
$$

We remark here that the statement of Theorem VI.3 can be used to obtain an estimate for the expected fraction of successful receivers without the conditioning on the event $A$. We write

$$
\begin{aligned}
\mathbb{E}^{(\mathbf{0}, \mathbf{1})}\left[\frac{R_{k, n}\left(G_{m}\right)}{\left|C_{\mathbf{0}}\left(G_{m}\right)\right|}\right]=\theta(\lambda) \mathbb{E}_{A}^{(\mathbf{0 , 1})}\left[\frac{R_{k, n}\left(G_{m}\right)}{\left|C_{\mathbf{0}}\left(G_{m}\right)\right|}\right]+ \\
(1-\theta(\lambda)) \mathbb{E}_{A^{C}}^{(\mathbf{0 , 1})}\left[\frac{R_{k, n}\left(G_{m}\right)}{\left|C_{\mathbf{0}}\left(G_{m}\right)\right|}\right] .
\end{aligned}
$$

Notice from Fig. 2 that $\theta(\lambda)$ shows a phase transition phenomenon. For the intensities we are interested in, $\mathbb{P}\left(A^{c}\right)=$ $1-\theta(\lambda)$ is very small and the latter term in the above equation can be neglected. This also suggests that Assumption 3 is not a very strong requirement.

Consequently, for large $m$, using Theorem VI.3 and Proposition VI.4 in (7) yields an approximation for the minimum forwarding probability given by,

$$
p_{k, n, \delta} \approx \inf \left\{p \mid \sum_{t=k}^{n-1} \frac{\theta_{k, t}^{\text {ext }}\left(\theta_{t, n}^{\text {ext }}-\theta_{t+1, n}^{\text {ext }}\right)}{\theta(\lambda)}+\frac{\theta_{k, n}^{\text {ext }} \theta_{n, n}^{\text {ext }}}{\theta(\lambda)} \geq 1-\delta\right\} .
$$

\section{Comparison with simulations}

We have not been able to obtain exact expressions for the probability $\theta_{k, t}^{\text {ext }}(\lambda, p)$ in terms of the percolation probability $\theta(\lambda)$. However, in Section VII-A, we provide some bounds

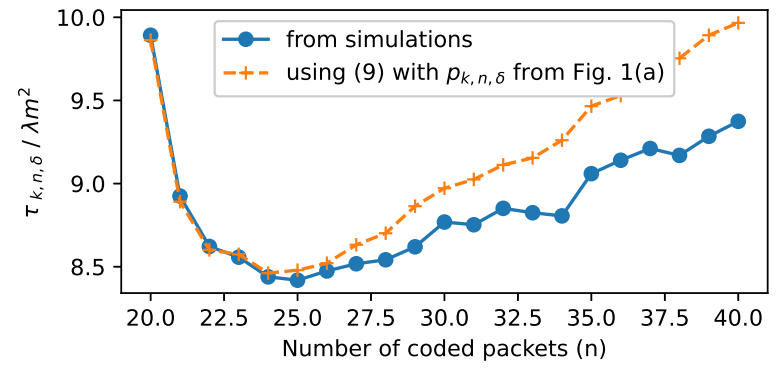

Fig. 3: Comparison of the expected number of transmissions per node in the RGG $(4.5,1)$ model on $\Gamma_{101}$ obtained using (9) with that obtained through simulations. Note that the $p_{k, n, \delta}$ value for each point on both the curves are from the simulations in Fig. 1(a).

for it. We also develop an alternate heuristic approach, which provides comparable results for the minimum forwarding probability obtained through simulations, in Section VII-B.

However, the approximation for the expected total number of transmissions, $\tau_{k, n, \delta}$ in (9) can be evaluated with the knowledge of the minimum forwarding probability. In Fig. 3, we plot $\tau_{k, n, \delta}$ normalized by $\lambda m^{2}$ with $n$, in which we use $p_{k, n, \delta}$ values from Fig. 1(a).

It is observed that for $n \lesssim 26$, both the curves match pretty well. However, for $n>26$ they diverge. This can be attributed to the fact that as $n$ increases, $p_{k, n, \delta}$ decreases as in Fig 1(a) and thus $\lambda p_{k, n, \delta} \searrow \lambda_{c}$. The estimate for the percolation probability, $\theta(\lambda)$, obtained via the ergodic result in (5) may not be accurate near the critical intensity, $\lambda_{c}$ (which is itself not exactly known). In particular, $\Gamma_{251}$ may not be large enough for the ergodic result in (5) to kick in, as we approach $\lambda_{c}$.

Nevertheless, this provides justification to our observation that the expected number of transmissions indeed decreases when we introduce coded packets along with probabilistic forwarding. This comes with a catch that the minimum forwarding probability for a near-broadcast behaves as in Fig 1(a). In order to establish this, we provide a heuristic explanation for it in the next section.

\section{DISCUSSION}

\section{A. Bounds on $\theta_{k, n}^{\text {ext }}(\lambda, p)$}

We give two lower bounds for $\theta_{k, n}^{\mathrm{ext}}(\lambda, p)$. The proofs for these are provided in the extended version of our paper [10].

A simple lower bound for $\theta_{k, n}^{\text {ext }}(\lambda, p)$ can be obtained by noting that if $\mathbf{0}$ is present in all $n$ IECs, then $\mathbf{0} \in C_{k, n}^{\text {ext }}$. Using the FKG inequality, we obtain

$$
\theta_{k, n}^{\mathrm{ext}}(\lambda, p) \geq \theta(\lambda p)^{n} .
$$

Note that this, along with Assumption 2, suffices to ensure that our analysis yields non-trivial results for all values of $k$ and $n$.

We now provide a second bound. Its derivation proceeds by constructing a new point process $\Phi_{T}$ with marks $Z_{T}=$ $\prod_{i \in T} Z_{i} \prod_{j \notin T}\left(1-Z_{j}\right)$, and relating the IEC of $\Phi_{T}$ to $C_{k, n}^{\text {ext }}$. We omit the details here. The lower bound obtained is

$$
\theta_{k, n}^{\text {ext }}(\lambda, p) \geq 1-\prod_{j=k}^{n}\left(1-\theta\left(\lambda p^{j}(1-p)^{n-j}\right)\right)^{\left(\begin{array}{c}
n \\
j
\end{array}\right)} .
$$




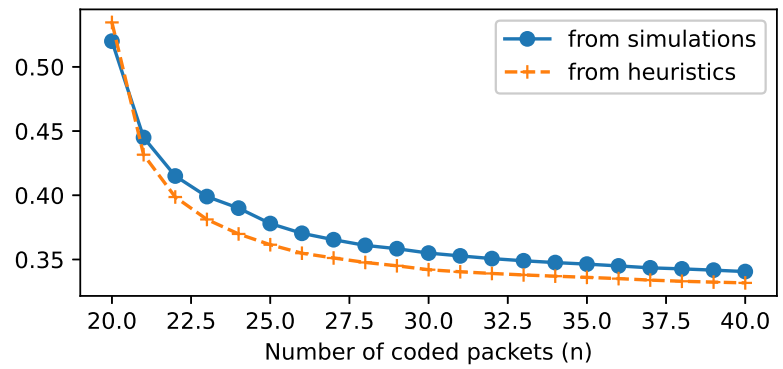

(a) Minimum retransmission probability

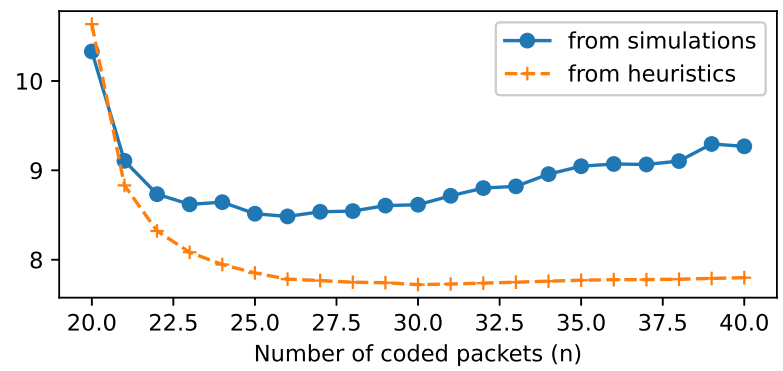

(b) Expected total number of transmissions

Fig. 4: Simulation results compared with results obtained using (14), (9) on $R G G(4.5,1)$ on $\Gamma_{101}$ with $k=20$ and $\delta=0.1$.

\section{B. A heuristic approach}

Motivated by the alternate interpretation for the nodes in the IEC expounded in Remark 3 of Section V, we provide a heuristic approach for evaluating the minimum forwarding probability. This is discussed in detail in [10].

As before, let $\theta^{\operatorname{ext}}(\lambda, p)$ denote the probability that the origin is in the IEC for a single packet transmission. Associate a new mark $\mathbf{Z}^{\prime}=\left(Z_{1}^{\prime}, Z_{2}^{\prime}, \cdots, Z_{n}^{\prime}\right) \in \mathbb{K}=\{0,1\}^{n}$ to each vertex of $\Phi$. The $i$-th coordinate of $\mathbf{Z}^{\prime}$ corresponds to probabilistic forwarding of the $i$-th packet. The mark $\mathbf{Z}^{\prime}$ is chosen such that each of the $i$ coordinates is either 1 with probability $\theta^{\mathrm{ext}}(\lambda, p)(=\theta(\lambda p))$ or 0 with the remaining probability, independent of the others. Similar to the viewpoint for the single packet transmission, our idea is to use $Z_{i}^{\prime}$ as a proxy for a vertex to be present in the IEC in probabilistic forwarding of the $i$-th packet. We refer to this as the mean-field model.

From the mean-field model, we obtain the following expression for the minimum forwarding probability:

$$
p_{k, n, \delta}^{\prime}=\inf \left\{p \mid \frac{\mathbb{P}(Y \geq k)}{\theta(\lambda)} \geq 1-\delta\right\}
$$

where $Y \sim \operatorname{Bin}\left(n,(\theta(\lambda p))^{2}\right)$. This is plotted in Fig. 4(a) and corresponding $\tau_{k, n, \delta}$ evaluated using (9) is plotted in Fig. 4(b). This behaviour is similar to what was obtained on the grid in [9]. While the $p_{k, n, \delta}^{\prime}$ curve tracks the simulations well, there is a minor difference that gets amplified when evaluating the expected total number of transmissions. This can be attributed to the drastic change in $\theta(\lambda)$ around the critical intensity $\lambda_{c}$.

\section{A note on our assumptions}

In this subsection, we provide some justifications for the assumptions made in our analysis. Our interest in this paper is to broadcast information on large and dense networks. A basic requirement for this is that a large number of nodes in the network must be reachable from the origin. In the sub- critical regime, i.e. $\lambda<\lambda_{c} \approx 1.44$, the clusters are finite and small. To model large dense ad-hoc networks, we need the graph to be connected on a large area $\Gamma_{m}$. This necessitates $\lambda$ to be in the super-critical regime and the component of the origin within $\Gamma_{m}$ to be large. In the limit as $m \rightarrow \infty$, this requires that the origin be present in the infinite cluster of the underlying RGG, thus justifying Assumption 3.

Further, notice that for a near-broadcast, we need the expected fraction of successful receivers to be close to 1 , i.e., $\mathbb{E}\left[\frac{\left|\mathcal{R}_{k, n}\left(\mathcal{G}^{\mathbf{0}}\right) \cap \Gamma_{m}\right|}{\lambda \theta(\lambda) m^{2}}\right] \geq 1-\delta$ for some small $\delta>0$ (The denominator here is the expected number of nodes within $\Gamma_{m}$ of the infinite cluster $C$.). If we would like this to hold for sufficiently large $m$, then the forwarding probability must be such that $\mathcal{R}_{k, n}\left(\mathcal{G}^{\mathbf{0}}\right)$ has infinite cardinality. This implies that $p$ must be such that there is an IEC during probabilistic forwarding on $\mathcal{G}^{0}$. Now, since existence of an IEC implies existence of an infinite cluster, the $p$ value must ensure presence of an infinite cluster. Thus $\lambda p>\lambda_{c}$. This justifies Assumption 2.

It can also be seen from the simulation results in Fig. 1 that $\tau_{k, n, \delta}$ is minimized when the forwarding probability is such that $\lambda p_{k, n, \delta}>\lambda_{c}$ or $p_{k, n, \delta}>0.32$. Further, results obtained from our heuristic approach in Fig. 4(a) and Fig. 4(b) also suggest that the expected total number of transmissions is indeed minimized when operating in the super-critical regime.

\section{REFERENCES}

[1] B. Williams and T. Camp, "Comparison of broadcasting techniques for mobile ad hoc networks," in Proc. 3rd ACM Int. Symp. Mobile Ad Hoc Networking \& Computing, 2002, pp. 194-205.

[2] C. Fragouli, J. Widmer, and J.-Y. Le Boudec, "Efficient broadcasting using network coding," IEEE/ACM Trans. Networking, vol. 16, no. 2, pp. 450-463, 2008.

[3] Y.-C. Tseng, S.-Y. Ni, Y.-S. Chen, and J.-P. Sheu, "The broadcast storm problem in a mobile ad hoc network," Wireless Networks, vol. 8, no. 2/3, pp. 153-167, 2002

[4] Y. Sasson, D. Cavin, and A. Schiper, "Probabilistic broadcast for flooding in wireless mobile ad hoc networks," in Proc. WCNC 2003, vol. 2, March 16-20, 2003, pp. 1124-1130.

[5] Z. J. Haas, J. Y. Halpern, and L. Li, "Gossip-based ad hoc routing," IEEE/ACM Trans. Networking, vol. 14, no. 3, pp. 479-491, 2006.

[6] F. Forero Rodríguez, "Geometric aspects and random delays in probabilistic broadcasting for wireless ad hoc networks," Ph.D. dissertation, Univ. Estadual de Campinas, Brazil, and Univ. de Los Andes, Colombia, 2019.

[7] R. Vaze, Random Wireless Networks. Cambridge Univ. Press, 2015.

[8] M. Franceschetti and R. Meester, Random Networks for Communication: From Statistical Physics to Information Systems. Cambridge Univ. Press, 2008.

[9] B. R. V. Kumar and N. Kashyap, "Probabilistic forwarding of coded packets on networks," IEEE/ACM Trans. Networking, vol. 29, no. 1, pp. 234-247, 2021.

[10] B. R. V. Kumar, N. Kashyap, and D. Yogeshwaran, "An analysis of probabilistic forwarding of coded packets on random geometric graphs," preprint, May 2021. [Online]. Available: arxiv.org/abs/2105.08779

[11] F. Baccelli and B. Błaszczyszyn, Stochastic Geometry and Wireless Networks, Volumes I and II. Now Publishers Inc, 2010.

[12] M. Penrose, Random Geometric Graphs. Oxford Univ. Press, 2003.

[13] J. Quintanilla, S. Torquato, and R. M. Ziff, "Efficient measurement of the percolation threshold for fully penetrable discs," Journal of Physics A: Mathematical and General, vol. 33, no. 42, p. L399, 2000.

[14] F. Baccelli, B. Błaszczyszyn, and M. K. Karray, Random Measures, Point Processes, and Stochastic Geometry. preprint, 2020. [Online]. Available: https://web.ma.utexas.edu/simons/wp-content/uploads/2020/ 02/BBK.pdf 